\title{
Impact of anesthetic technique in endobronchial ultrasound bronchoscopy: patient and operator satisfaction
}

\author{
Flintras D.1, Gioni P.1, Emmanouil P.2, Koufos N.2, Stratakos G.2, Dragini G.1 \\ 1 Mediterraneo Hospital, Dept of Anaesthesiology, Athens, Greece, 2Mediterraneo \\ Hospital, Interventional Pulmonology, Athens, Greece
}

Background and Goal of Study: EBUS bronchoscopy is widely recognized as a valuable tool for staging of lung cancer thus diminishing dramatically the need of surgical approach ${ }^{1}$. Takes significantly longer than conventional bronchoscopy and may therefore cause more discomfort. Anaesthetic technique is of paramount importance regarding patient and bronchoscopist satisfaction and quality of procedure. We aimed to describe the impact of anaesthetic technique in achieving safe and comfortable procedure for the patient and an easy and successful procedure for the bronchoscopist.

Materials and Methods: Fifty seven patients, undergoing EBUS bronchoscopy under GA with laryngeal mask (i-Gel supraglottic mask), were studied. For this procedure we used pre-operatively Midazolam as anxiolytic, TIVA (Propofol/Remifentanil infusion) intraoperatively with small dose of short acting muscle relaxant (Atracurium). Postbronchoscopy, patients responded to a Visual Analogue Scale (VAS) questionnaire for tolerability of bronchoscopy and acceptance to repeat the procedure. Bronchoscopist responded to a Visual Analogue Scale (VAS) questionnaire for quality of bronchoscope handling and easiness of the procedure. The frequency of coughing and desaturation and the length of the procedure and recovery time was recorded.

Results and Discussion: This anaesthetic technique revealed some outstanding results. 57 patients $(100 \%)$ reported that they couldn't recall any distressing symptoms, and they would return for the procedure if required. Interventional bronchoscopist satisfaction was excellent in $100 \%$ of the cases. Supraglottic device (iGel) unique properties provide excellent environment for the anaesthetist and bronchoscopist. Low dose short acting muscle relaxant eliminate cough and motion, while there was no incidence of desaturation. Duration of the procedure was $72 \mathrm{~min}$ in average. Ultra-short acting medication (Propofol/Remifentanil infusion) reduces time interval from the end of the procedure till the removal of the laryngeal mask, which was less than $3 \mathrm{~min}$.

Conclusion(s): G.A. with the use of supraglottic mask, TIVA (Propofol/Remifentanil infusion), and small dose of short acting muscle relaxant is associated with outstanding patient and bronchoscopist satisfaction, improves the quality of the procedure by eliminating common problems (cough, desaturation, difficulty in bronchoscope handling) and reduces recovery time to minimum.
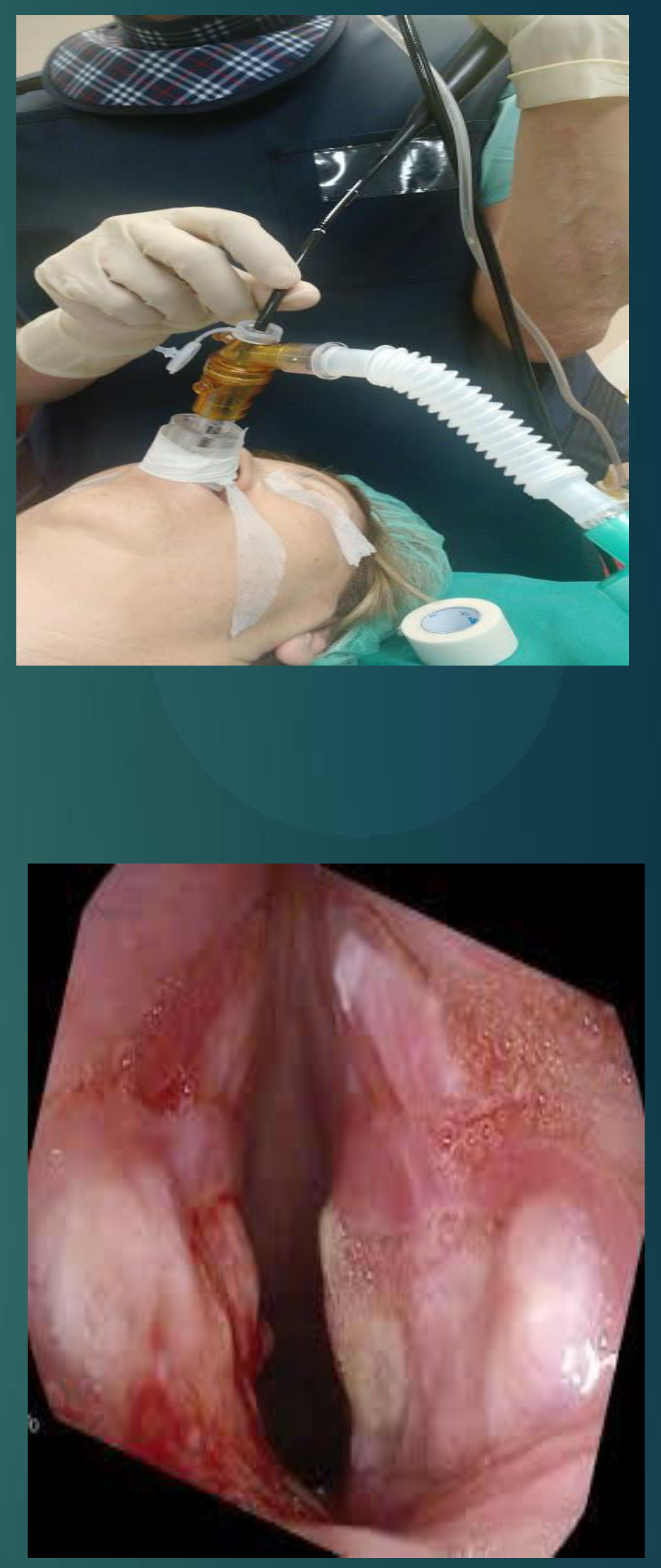

References: 1. Imaging in Clinical Oncology, Gouliamos A. pp 189 